\section{Hepatocellular carcinoma and African iron overload}

EDITOR,-Gangaidzo and Gordeuk (Gut 1995; 37: 727-30) postulate that iron overload may be a risk factor for hepatocellular carcinoma (HCC) - a subject of obvious interest to workers in Africa. It is especially so to one of us (AW) who, originally, in 1953, propounded the iron overload hypothesis. This sought to explain the abnormal iron deposition (siderosis) present in many Africans, caused by a high adventitious intake of the element arising from food preparation in iron utensils. ${ }^{1}$

In comment, firstly, the above authors state that HCC is probably the commonest malignancy occurring in men worldwide. Actually it ranks eighth in numerical importance on a worldwide basis, accounting for $5 \cdot 3 \%$ of new cancers in men and $2 \cdot 6 \%$ in women. ${ }^{2}$

Next, it is insufficiently appreciated that its incidence in men in Africa is highly variable, ranging from 47.9 per 100000 world population, in Mali, ${ }^{3}$ to 7.5 in Uganda, ${ }^{4}$ and 6.4 in South Africa. ${ }^{5}$ In agreement with the latter, recently, in three rural widely separated hospitals, responsible for the needs of about 400000 Africans, we found an average of five admissions of men for HCC per 100000 annually. Interestingly, the latter rates are much the same as those of Afro-Americans, 5.0-6.6 per $100000 .{ }^{3}$ The citing of 100 cases per 100000 in Mozambique is inapposite as no current study of the painstaking type made in Uganda, ${ }^{4}$ has been pursued in Mozambique - a country at war for 20 years. We know of no present day excessively high rates for HCC in African countries south of the Equator.

Turning now to the noxiousness of iron overload, in the original hypothesis, ${ }^{1}$ also in a subsequent local study based on 296 postmortem examinations at Baragwanath Hospital, Soweto, Johannesburg, ${ }^{6}$ the pathogenicity of iron overload was doubted, for no constant correlation was found between the degrees of fibrosis and cirrhosis and the amount of iron pigment in the liver. However, later, at the same hospital, as recently detailed by Lynch, ${ }^{7}$ associations were reported of siderosis with osteoporosis, diabetes, oesophageal cancer, and heart failure. The findings in these studies, however, made over 20 years ago, have not been currently confirmed. More to the point, over the decades, no attempt has been made at the village level to learn whether iron overload contributes significantly to morbidity and mortality; that is, is it disadvantageous to 1 per 100 , or 1 per 1000 ?

In an investigation made in 1985 by Gordeuk $e a^{8}{ }^{8}$ in Zimbabwe, it was estimated that there were about 80000 cases of severe iron overload. In the same year, it was stated that in that country there were 14587 cases of malaria, 2956 of tuberculosis, and 144 of cholera. Surely, were iron overload as pathognomonic as is conjectured, there should be a plethora of evidence incriminating it. This does not seem to be the case.

Gangaidzo and Gordeuk regret that in Africa, only about half of the cases of HCC are explicable on the basis of $\mathrm{HBV}$ infection. It must be recognised that apart from the cause and effect of classic deficiency diseases, the above proportion or less is common with multifactorial diseases - dental caries, coronary heart disease, and certain cancers. In brief, the balance of knowledge and ignorance regarding the causation of HCC is the usual.
We entirely support the authors in their urge that prospective studies should be undertaken to examine the possible role of African overload in the pathogenesis of HCC. However, at the village level, in the requisite studies, major difficulties will be encountered regarding participation, especially regarding blood taking and the fear of AIDS. A complicating factor for HCC, as Kew and associates ${ }^{9}$ have shown, is that the infection rate of HBV, an important aetiological factor, varies not only from region to region, but from village to village. Additionally, there will be the usual difficulties in seeking to resolve whether iron overload, if implicated, is a causative or an associated factor. Not least of perplexities is the situation whereby a given parameter, for example, serum ferritin concentration, can have a differing significance according to the context. As a recent example of this phenomenon, at Belfast and Toulouse, there is the same average serum cholesterol value but a fourfold difference in mortality from coronary heart disease. ${ }^{10}$

Finally, should iron overload be meaningfully incriminated, the authors say it is eminently preventable through changing the practices of preparation and consumption of traditional beer, and it is treatable by phlebotomy therapy to remove iron from the body'. In our view, both of the suggested procedures, in the rural context, are non-starters.

To reiterate, while we are unconvinced of the clinical importance of iron overload, the issue must be resolved - that is, whether it is or is not of public health significance within the context of impoverished Africa.

A R P WALKER Human Biochemistry Research Unit, Department of Tropical Diseases, School of Pathology of the

University of the Witwatersrand, and the South African Institute for Medical Research, Fohannesburg,

ISIDOR SEGAL Gastroenterology Unit,
Baragwanath Hospital University of the Witwatersrand, Johannesburg, Walker ARP, Arvidsson UB. Iron 'overoad' in Med Hyg 1953; 47: 536-48.

2 Tomatis L. Cancer: causes, occurrence and control. International Agency for Research on Cancer, IARC Scientific Publications No 100. Lyon: World Health Organization, 1990: 59.

3 Parkin BM, Muir CS, Whelan SL, et al. Cancer incidence in five continents. Vol 6. Lyon: IARC, 1992: 930

4 Wabinga HR, Parkin DM, Wabwire-Mangen F, Mugerwa JW. Cancer in Kampala, Uganda, in 1989-91: changes in incidence in the era of 1989-91: changes in incidence in

5 Sitas F, Pacella R. Cancer registry of South Africa, 1989. Johannesburg: South African Institute for Medical Research, 1994: 33.

6 Higginson J, Gerritsen Th, Walker ARP. Siderosis in the Bantu of Southern Africa. Am f Pathology 1953; XXIX: 779-815.

7 Lynch SR. Iron overload: prevalence and impact on health. Nutr Rev 1995; 53: 255-60.

8 Gordeuk VR, Boyd RD, Brittenham GM. Dietary iron overload persists in rural subSaharan Africa. Lancet 1986; i: 1310-3.

$9 \mathrm{Kew}$ MC. Chronic hepatitis B virus infection and hepatocellular carcinoma in Africa. $S A f r \mathcal{F}$ Sci 1992; 88: 524-8.

10 Evans AE, Ruidavets J-B, McCrum EE, et al. Autres pays, autres coeurs? Dietary patterns,
risk factors and ischaemic heart disease in Belfast and Toulouse. Qf Med 1995; 88: 469-77.

\section{Reply}

EDITOR,-We thank Drs Walker and Segal for their perspectives on the prevalence of hepatocellular carcinoma (HCC) and the toxicity of iron overload in Africa.

High quality cancer epidemiological data are difficult to come by in Africa. With regard to the estimate of Drs Walker and Segal of five admissions for HCC per 100000 population annually in rural South Africa, we note that hospital based studies may be unreliable estimates of countrywide incidence rates. ${ }^{1}$ $\mathrm{HCC}$ is a common and important condition in Africa. At least one authority has observed, with regard to HCC, that 'some of the documented incidences are almost certainly underestimates, perhaps by as much as 50 percent in some countries'. ${ }^{2}$ A contemporary textbook of gastroenterology in the tropics offers an adjusted rate for HCC of 113 per 100000 per annum in Shangaan men inhabiting the border areas of Mozambique, Zimbabwe, and South Africa. ${ }^{2}$ In Harare, Zimbabwe, HCC is the most common malignancy in men in the 1990s (Harare Cancer Registry, 1995). In the internal medicine wards of one of us (ITG) at Harare Central Hospital, HCC was the most common cancer diagnosis in men in 1993-1994 and accounted for $3 \cdot 2 \%$ of admissions. Among those of us who regularly provide medical care to rural Africans (constituting more than $80 \%$ of the Zimbabwean population), there is general agreement that $\mathrm{HCC}$ represents a major health problem. We have seen 'few more depressing tasks than caring for a patient with this particular malignancy, ... and there is no satisfactory treatment'.3

The question of whether iron overload in Africa is 'noxious', seems to recapitulate the discussion of 30 years ago about whether iron overload is damaging in people of European origin with hereditary haemochromatosis. As we learn more about states of excess iron, it is our opinion that the weight of evidence points to the conclusion that iron overload of whatever cause (HLA linked hemochromatosis, transfusions, ineffective erythropoiesis, or high dietary iron in Africa) is potentially toxic and that medical practitioners are obligated to work to prevent and treat iron overload in their patients. ${ }^{4}$ In a series of careful pathological studies 35 years ago, Professor T H Bothwell and colleagues at the University of the Witwatersrand provided convincing evidence that dietary iron overload directly causes cirrhosis. ${ }^{5-7}$ Interestingly, in 1960 Bothwell reported that in Africans the hepatic iron threshold above which portal fibrosis and cirrhosis are likely to be present is a concentration of $360 \mu \mathrm{mol} / \mathrm{g}$ dry weight ${ }^{5}$ (normal is less than $30 \mu \mathrm{mol} / \mathrm{g}$ dry weight). Twenty six years later Dr L Powell's group in Australia reported an almost identical threshold for hepatic damage among white subjects with hereditary hemochromatosis. ${ }^{8}$ Support for the association between African iron overload and cirrhosis is provided by a study conducted in the late 1980 s at a mission hospital in Swaziland: nine of 29 consecutive adults undergoing diagnostic liver biopsy had hepatic iron concentrations over $360 \mu \mathrm{g} / \mathrm{g}$ dry weight and seven of these subjects $(78 \%)$ had either portal fibrosis or cirrhosis on histological examination. ${ }^{9}$

Two recent studies that we have conducted examine the noxiousness of dietary iron overload in data sets that span the historical spectrum of this disease. Firstly, we analysed data from the original study of iron overload in Africans, conducted by Dr A S Strachan between 1925 and 1928 at Johannesburg General Hospital, to determine if this form of iron loading may be associated with deaths from HCC or tuberculosis. ${ }^{10}$ In the original 
study, necropsies were performed on 604 adult blacks from southern Africa and one of five grades of hepatic and splenic iron was assigned based on the Prussian blue reaction obtained when a piece of tissue was dipped in a mixture of potassium ferrocyanide and hydrochloric acid. ${ }^{11}$ Using logistic regression models, we found that iron overload was strongly associated with the findings of cirrhosis $(p<0.0001)$, HCC $(p=0.0002)$, and tuberculosis $(p<0.0001) .10$ Secondly, we reviewed all 320 diagnostic liver biopsy specimens processed at the University of Zimbabwe from 1992 to 1994 . HCC was present in $19 \%$ of the evaluable specimens, cirrhosis in $21 \%$, and high grades of iron in $19 \%$. We found significant associations between the presence of iron overload and the histological diagnoses of cirrhosis and HCC.

We do not completely share the pessimism of Drs Walker and Segal with regard to changing the method of preparation of traditional beer and to instituting a phlebotomy programme. The iron drums that are now used to prepare traditional beer replaced clay cooking pots around the turn of the century, but these clay utensils are still used in most rural communities to prepare food and other forms of beverages. It seems feasible to us to encourage the use of these clay pots for the preparation of traditional beer in place of the newer and more convenient, but probably more dangerous, iron drums. For the past two years our research team has conducted a rural based study of dietary iron overload. We have been struck by the overwhelming level of cooperation that we have been able to obtain through close and regular contact with the rural communities. Despite the need for venesection and a high awareness of the problem of HIV, it has been unusual for subjects to refuse to take part.

In summary, we believe the available data point to both HCC and dietary iron overload as major heath problems in rural Africa. There is a strong body of evidence to suggest an association between the two conditions. Major initiatives are needed to combat these diseases beginning in the communities where the people live.

INNOCENT TICHAONA GANGAIDZO Department of Medicine and the University of Zimbabwe School of Medicine, PO Box A178, Avondale,

Harare,

Zimbabwe

VICTOR ROLAND GORDEUK Department of Medicine,
George Washington University Medical Center,
Washington DC, $U S A$

1 Sackett DL, Hayes RB. Bias in analytic research. f Chron Dis 1979; 32: 51-63.

2 Kew MC. Hepatocellular carcinoma: epidemiology. In: Watters D, Kiire CF, eds. ogy. In: Watters D, Kiire CF, eds.
Gastroenterology in the tropics and subtropics. Gastroenterology in the tropics and
London: Macmillan, 1995: 184-6.

3 Cook GC. Hepatocellular carcinoma: one of the world's most common malignancies. Q F Med 1985; 57: 705-8.

4 Gordeuk VR, McLaren G, Samowitz W. Etiologies, consequences and treatment of iron overload. Crit Rev Lab Clin Med 1994; 31: 89-133.

5 Bothwell TH, Bradlow BA. Siderosis in the Bantu. A combined histopathological and chemical study. Arch Pathol 1960; 70: 279-92.

6 Bothwell TH, Isaacson C. Siderosis in the Bantu. A comparison of incidence in males and females. BMF 1962; i: 522-4.

7 Isaacson C, Seftel HC, Keeley KJ, Bothwell TH. Siderosis in the Bantu: the relationship between iron overload and cirrhosis. $\mathcal{F} \mathrm{Lab}$ between iron overload and
Clin Med 1962; 58: 845-53.

8 Bassett ML, Halliday JW, Powell LW. Identification of homozygous hemochromatosis subject by measurement of hepatic iron sis subject by measurement of
index. Hepatology 1986; 6: 24-9.
9 Friedman BM, Baynes RD, Bothwell $T H$ Gordeuk VR, MacFarlane BJ, Lamparelli RD, et al. Dietary iron overload in southern African et al. Dietary iron overload in southern
blacks. S Afr Med f 1990; 78: 301-5.

10 Gordeuk VR, MacPhail AP, McLaren CE Deichsel G, Bothwell TH. Associations of iron overload in Africa with hepatocellular carcinoma and tuberculosis: Strachan's 1929 thesis revisited. Blood 1996; 87: 3470-6.

11 Strachan AS. Haemosiderosis and haemochroma tosis in African natives with a comment on the aetiology of haemochromatosis. [MD thesis]. Glasgow: University of Glasgow, 1929.

\section{Analysis of biological variables in Crohn's disease}

EDITOR,-We wish to comment on the paper by Sahmoud et al (Gut 1995; 37: 811-8) where the authors suggested the following features: age, duration of disease, interval since previous relapse, and colonic involvement as powerful prognostic factors to predict relapse in quiescent Crohn's disease. We also followed up for 18 months 107 patients with Crohn's disease in clinical remission ${ }^{1}$ and we used laboratory tests enhanced by clinical characteristics for predicting relapse.

Interestingly, our results about clinical characteristics were, for some aspects, similar to those obtained by the French group. A Cox regression analysis indicated interval since previous relapse, previous surgery, location (ileum versus non-ileum), and occurrence of postsurgical clinical recurrence as predictors of relapse; however an intercorrelation was found among these characteristics and particularly between previous surgery and location (but simultaneous Cox regression indicated that surgery would be more predictive than location). Thus two groups of risk of relapse according to clinical characteristics were defined: (a) favourable group consisted of patients in clinical remission after last relapse for at least 24 months or patients with less than 24 months but who had undergone surgery without subsequent post surgical clinica recurrence; (b) unfavourable group consisted of patients in clinical remission for less than 24 months not included in the favourable group.

In the French study surgery was not identified as a prognostic factor; this could be explained because in that study quiescent disease induced by surgery seems to be excluded and ileal location (and for this reason also previous resection) is present in a small number of patients in comparison with colonic location. In our experience duration of illness was not a useful predictor of relapse; we did not study age of patients.

In the study by Sahmoud et al biological indicators such as albumin and erythrocyte sedimentation rate did not result in predicting the course of the disease; in contrast, in our experience, laboratory tests seem to be extremely useful for this aim. In a previous study $^{2}$ a prognostic index based on laboratory tests was proposed. In our last study ${ }^{1}$ the ability of this index to predict relapse was validated and a simplified application was proposed; patients with at least one of the laboratory tests changed ( $\alpha_{1}$ acid glycoprotein $>130 \mathrm{mg} / \mathrm{dl}$, or $\alpha_{2}$ globulin $>9.0 \mathrm{gm} / 1$ or erythrocyte sedimentation rate $>40 \mathrm{~mm} / \mathrm{h}$, or all three) presented within 18 months a relapse rate of $75 \%$ whereas in patients with normal tests it was $13 \%$. In our study nine among 18 false-negative (normal laboratory tests with subsequent relapse) were represented by patients with previous remission $<3$ months who had recently stopped corticosteroid treatment. Usually, however, values of laboratory tests are rapidly normalised by a period of corticosteroid treatment and it is shown ${ }^{34}$ that these tests, performed during or immediately after corticosteroids have poor ability in discriminating the clinical outcome. In our opinion the French investigators were unable to show a prognostic ability of sedimentation rate and albumin because at least 64 patients among 167 included were treated with corticosteroids at the entry of the study (placebo group of the trial about usefulness of prednisolone treatment in patients in clinical remission after prednisolone treatment for an acute attack).

We agree, however, with Sahmoud's conclusions about the necessity of identifying patients with different risks of relapse in quiescent Crohn's disease. In our study, at the end of 18 months of follow up, the predicted proportion of patients with relapse was $13 \%$ and $31 \%$ in the subgroup with normal laboratory tests and favourable and unfavourable clinic characteristics respectively, and $87 \%$ in patients with abnormal tests and unfavourable characteristics. Thus risk of relapse varies substantially among different subgroups of patients with clinical remission; prediction of this risk is helpful not only for a correct design of clinical trials, but also, in clinical practice, for timing subsequent clinical visits and for selecting groups of patients where preventive treatment could be justified.

C BRIGNOLA C BELLOLI MPIER P GIONCHETTI A BELLUZZI astroenterologia, Policlinico $S$ Orsola,, ,
Massarenti 9 Massarenti 9,
40138 Bologna, Italy

1 Brignola C, Iannone P, Belloli C, De Simone G, Bassein I, Gionchetti P, et al. Prediction of relapse in patients with Crohn's disease in remission: a simplified index using laboratory tests, enhanced by clinical characteristics. European fournal of Gastroenterology and Hepatology 1994; 6: 955-61.

2 Brignola C, Campieri M, Bazzocchi G, Farruggia P, Tragnone A, Lanfranchi GA. A laboratory $\mathrm{P}$, Tragnone A, Lanfranchi GA. A laboratory
index for predicting relapse in asymptomatic patients with Crohn's disease. Gastroenterology 1986; 91: 1490-4.

3 Landi B, N'Guyen Anh T, Cortot A, Soule JC, Rene E, Gendre JP, et al. Endoscopic monitoring of Crohn's Disease treatment: a prospective, randomized clinical trial. Gastroenterology 1992; 102: 1647-53.

4 Brignola C, De Simone G, Belloli C, Iannone P, Belluzzi A, Gionchetti $P$, et al. Steroid treatment in active Crohn's disease: a comparison between two regimens of different duration. Aliment Pharmacol Ther 1994; 8: 465-8.

\section{Reply}

EDITOR,-Thank you for referring the interesting comments of Brignola et al to us concerning our article. The Italian group has considerable experience in investigating the value of biological parameters in Crohn's disease and has published several research results in that field. One of their earliest publications ${ }^{1}$ suggested a prognostic index for predicting relapse in quiescent Crohn's disease patients using the baseline blood values of $\alpha_{1}$ glycoprotein, $\alpha_{2}$ globulin, and the erythrocyte sedimentation rate. This was based on data from 41 patients who had been in remission for at least six months. The same study suggested that the blood values of C-reactive protein and $\alpha_{1}$ antitrypsin were of borderline significance and the predictive value of some other biological variables were much less important, namely the haemoglobin concentration, the white blood cell count, serum iron, albumin, and $\gamma$ globulin blood values. 